\title{
Publisher Correction: Atherogenic dyslipidemia promotes autoimmune follicular helper T cell responses via IL-27
}

Heeju Ryu, Hoyong Lim, Garam Choi, Young-Jun Park, Minkyoung Cho, Hyeongjin Na, Chul Won Ahn, Young Chul Kim, Wan-Uk Kim, Sang-Hak Lee and Yeonseok Chung (D)

Correction to: Nature Immunology https://doi.org/10.1038/s41590-018-0102-6, published online 30 April 2018.

In the version of this article initially published, the third label along the horizontal axis of Fig. $4 \mathrm{~b}$ (Il13a) and the middle label above each plot in Fig. 6k $\left(\mathrm{Stat}^{--}\right)$were incorrect, and the hash marks along the horizontal axis for Fig. 6i were spaced incorrectly. Also, the statistical results in the citation for Supplementary Fig. 5a $\left({ }^{\star} P<0.05,{ }^{* *} P<0.01\right.$ and ${ }^{* *} P<0.001$ (unpaired Student's t-test)) in the fifth subsection of Results were incorrect. The correct label for Fig. $4 \mathrm{~b}$ is $I l 23 a$ and for Fig. $6 \mathrm{k}$ is Stat $1^{-1}$, and the right hash mark along the horizontal axis for Fig. 6i should be beneath the data points at right. The correct citation of the statistical results is as follows: " $P<0.05$ and $P<0.01$ (unpaired Student's $t$-test); Supplementary Fig. 5a)." The errors have been corrected in the HTML and PDF version of the article.

Published online: 19 June 2018

https://doi.org/10.1038/s41590-018-0158-3

\section{Publisher Correction: The transcriptional coactivator TAZ regulates reciprocal differentiation of $T_{H} 17$ cells and $T_{\text {reg }}$ cells}

Jing Geng, Shujuan Yu, Hao Zhao, Xiufeng Sun, Xun Li, Ping Wang, Xiaolin Xiong, Lixin Hong, Changchuan Xie, Jiahui Gao, Yiran Shi, Jiaqi Peng, Randy L Johnson, Nengming Xiao, Linrong Lu, Jiahuai Han, Dawang Zhou and Lanfen Chen

Correction to: Nature Immunology 18, 800-812 (2017); https://doi.org/10.1038/ni.3748; published online 15 May 2017.

In the version of this article initially published, the institution name for affiliation 3 (Maryland Anderson Cancer Center) was incorrect. The correct institution is MD Anderson Cancer Center. The error has been corrected in the HTML and PDF versions of the article.

Published online: 14 February 2018

https://doi.org/10.1038/s41590-018-0055-9

\section{Publisher Correction: Transcription factor Hoxb5 reprograms B cells into functional T lymphocytes}

Mengyun Zhang, Yong Dong, Fangxiao Hu, Dan Yang, Qianhao Zhao D, Cui Lv, Ying Wang, Chengxiang Xia, Qitong Weng, Xiaofei Liu, Chen Li, Peiqing Zhou, Tongjie Wang, Yuxian Guan, Rongqun Guo, Lijuan Liu, Yang Geng, Hongling Wu, Juan Du (D), Zheng Hu, Sheng Xu, Jiekai Chen, Aibin He, Bing Liu, Demin Wang, Yong-Guang Yang and Jinyong Wang (D)

Correction to: Nature Immunology https://doi.org/10.1038/s41590-018-0046-x, published online 12 February 2018.

In the version of this article initially published, some identification of the supplementary information was incorrect. The items originally called Supplementary Tables 1, 2, 3, 4 and 5 should be Source Data Figures 1, 2, 4, 5 and 7, respectively; those originally called Supplementary Tables 6, 7 and 8 should be Supplementary Tables 1, 2 and 3, respectively; and those originally called Source Data Figures 1, 2, 4, 5 and 7 should be Supplementary Tables 4, 5, 6, 7 and 8, respectively. The errors have been corrected in the HTML version of the article. 